\title{
REMOCIÓN DE MATERIA ORGÁNICA EN REACTOR ANAEROBIO DE MANTO DE LODOS DE FLUJO ASCENDENTE EN EL TRATAMIENTO DE AGUAS RESIDUALES DEL CAMAL DE HUANCAVELICA
}

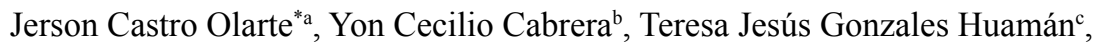 \\ Liliana Sumarriva Bustinza ${ }^{\mathrm{d}}$
}

\begin{abstract}
RESUMEN
En la presente investigación se evaluó un nuevo sistema de tratamiento primario para aguas residuales industriales, se utiliza el "Reactor anaerobio de manto de lodos de flujo ascendente" (UASB), aplicado a la remoción de materia orgánica. El sistema a escala piloto fue diseñado para las aguas residuales del Camal Municipal de Huancavelica, el cual se bombeó después del pre tratamiento (cámara de reja gruesa y desarenador) al tanque de almacenamiento de 350 litros para realizar el tratamiento del agua residual en el reactor UASB a escala laboratorio. Se evaluó la eficiencia de remoción de materia orgánica en un reactor UASB, para un tiempo de retención hidráulico (TRH) de 14 horas con un caudal de circulación de $35 \mathrm{~mL} / \mathrm{min}$ se obtuvo una eficiencia mínima de remoción de Demanda Química de Oxígeno (DQO) de 33,66 \% y una máxima eficiencia de 76,90\% todo ello en un rango de temperatura de agua residual de $10^{\circ} \mathrm{C}$ y $19^{\circ} \mathrm{C}$. También para este TRH de 14 horas la cantidad de sólidos suspendidos totales disminuyó de $524,34 \mathrm{mg} / \mathrm{L}$ a $365,04 \mathrm{mg} / \mathrm{L}$, obteniéndose una eficiencia de remoción de sólidos suspendidos totales de 30,28\%. El tratamiento primario reactor UASB es una alternativa para remover la materia orgánica y sólidos suspendidos totales, aplicado para aguas residuales del Camal Municipal de Huancavelica y en localidades con condiciones de clima y altitud similares; el diseño se optimizó para un TRH de 14 horas para el reactor UASB el cual elevó la eficiencia de remoción de materia orgánica
\end{abstract}

Palabras clave: reactor UASB, tratamiento anaerobio, tiempo de retención hidráulico, carga orgánica.

\footnotetext{
a Facultad de Ciencias de Ingeniería, Instituto de Investigación, Universidad Nacional de Huancavelica. Avenida Agricultura 319-321, Paturpampa, Huancavelica, Perú. Email: *jersoncastro2907@gmail.com

b Ingeniero Consultor

c Universidad Nacional de Huancavelica, Perú.

¿ Departamento de Química, Facultad de Ciencias, Universidad Nacional de Educación "Enrique Guzmán y Valle".
} 


\title{
REMOVAL OF ORGANIC MATTER IN AN UPFLOW ANAEROBIC SLUDGE BLANKET REACTOR IN THE TREATMENT OF WASTEWATERIN A SLAUGHTERHOUSE IN HUANCAVELICA
}

\begin{abstract}
This research evaluated a new system of primary treatment for industrial waste water, an"Anaerobic Upflow sludge blanket Reactor" (UASB) was used for the removal of organic matter. The pilot scale system was designed for the wastewater of the Municipal Slaughterhouse in Huancavelica, which was pumped after the pre-treatment (coarse screen and grit removal) to the storage tank of 350 liters for later to perform the treatment of wastewater in UASB reactor scale laboratory. The removal efficiency of organic matter in an UASB reactor was evaluated, for a hydraulic retention time (HRT) of 14 hours with a flow rate of $35 \mathrm{~mL} / \mathrm{min}$, the minimum efficiency of Chemical Oxygen Demand (COD) removal was $33.66 \%$ and maximum efficiency was $76.90 \%$ in a temperature range of wastewater from $10{ }^{\circ} \mathrm{C}$ to $19{ }^{\circ} \mathrm{C}$. Also for this HRT of 14 hours the amount of total suspended solids decreased from $524.34 \mathrm{mg} / \mathrm{L}$ to $365.04 \mathrm{mg} / \mathrm{L}$, obtaining a removal efficiency of total suspended solids of $30.28 \%$. The UASB reactor primary treatment is an alternative to remove the organic matter and total suspended solids applied for wastewater from the Municipal Slaughterhouse of Huancavelica and cities situated in places with similar climate and altitude; the design was optimized for a HRT of 14 hours for the UASB reactor, which increased the efficiency of organic matter removal.
\end{abstract}

Key words: UASB reactor, anaerobic treatment, hydraulic retention time, organic load.

\section{INTRODUCCIÓN}

La Universidad Nacional de Huancavelica, como universidad pública, recibe recursos financieros provenientes del Fondo de Desarrollo Socioeconómico del Proyecto Camisea "FOCAM", destinados exclusivamente a la investigación científica, tecnológica e innovación.

El Camal Municipal de Huancavelica, ubicado en el distrito de Huancavelica; centro poblado: Paltarumi - Chuñuranra, a una altitud de $3715 \mathrm{msnm}$, cuenta con una planta de tratamiento de agua residual, la cual tiene las siguientes operaciones y procesos unitarios como: cribado, desarenador, trampa de grasa, tanque séptico, sedimentadores, lecho de secado y como último el humedal artificial. El sistema de tratamiento presenta deficiencia en el diseño de las operaciones y procesos unitarios como: dimensionamiento incorrecto del cribado, desarenador, tanque séptico, sedimentador, lecho de secado y humedal artificial.

En este sistema de tratamiento, desde que empezó a operar en el año 2011, no se realizó el mantenimiento respectivo, y el monitoreo del agua tratada. Como resultado del diagnóstico realizado se pudo observar que el efluente del tratamiento tiene un color gris oscuro; el color 
del efluente es un indicador que el sistema de tratamiento es ineficiente.

Como consecuencia del vertido sin tratamiento al ambiente del agua residual del camal municipal de Huancavelica, se contaminan los suelos y los cuerpos de agua, además de incrementar el riesgo de la salud por la proliferación de malos olores producto de la degradación natural de la materia orgánica.

Por ello, la investigación científica realizada busca alternativas técnicas de solución de bajo costo a la problemática del tratamiento, disposición y la reutilización inadecuada de las aguas residuales del Camal Municipal de Huancavelica.

Dado al alto contenido de materia orgánica biodegradable presente en el agua residual de camales municipales, se utilizó la tecnología del reactor UASB ya que los procesos anaerobios suelen ser técnicas viables y económicamente más factibles para su tratamiento.

Los métodos de tratamiento del agua residual en mataderos se pueden dividir en dos grupos: anaerobios y aerobios. Los tratamientos anaerobios permiten un alto grado de reducción de la DBO, producen una menor cantidad de lodos y permiten la obtención de biogás que puede ser reutilizado en el propio matadero; los tratamientos aerobios ayudan a la transformación del nitrógeno orgánico y amoniacal en nitrato y no generan fuertes olores como los anaerobios ${ }^{1}$.

La ventaja de los reactores UASB es que los costos de operación y mantenimiento son relativamente bajos. El reactor UASB es empleado para tratar aguas residuales industriales y domésticas. La investigación tuvo como objetivo evaluar la eficiencia de remoción de materia orgánica en un Reactor Anaerobio de Manto de Lodos de Flujo Ascendente (UASB) para el tratamiento de aguas residuales del Camal Municipal de Huancavelica.

\section{Muestra}

\section{PARTE EXPERIMENTAL}

El tipo de muestra es no probabilística, por ello se trabajó con caudales de 50L/día y 58L/día. Estos caudales se determinaron porque el reactor UASB tiene un volumen útil de 29 litros y los tiempos de retención hidráulico a investigar fueron de 14 y 12 horas.

\section{Equipo experimental}

Para realizar el estudio se utilizó un reactor UASB construido de polietileno, con capacidad de 29 litros. El reactor UASB se instaló en el Camal Municipal de Huancavelica, se inoculó con lodo anaerobio (en un $30 \%$ del volumen útil) proveniente del CITRAR-UNI. En el fondo del reactor se colocó un aproximado de 20 canicas, para proveer una distribución uniforme del flujo.

Respecto a otro tipo de la distribución uniforme. En el fondo del reactor se colocó grava para proveer una distribución uniforme del flujo, la que ocupó aproximadamente un $4 \%$ del volumen útil del reactor ${ }^{2}$. 


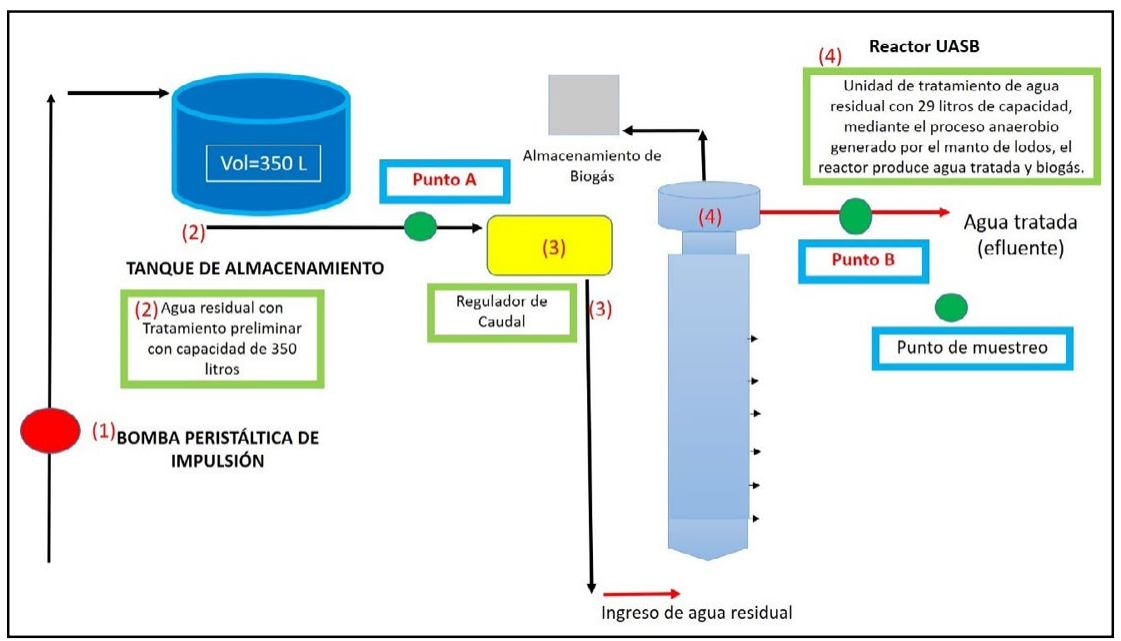

Figura 1. Esquema general de la investigación.

Las aguas residuales industriales del Camal Municipal de Huancavelica, luego de un pretratamiento (cámara de rejas gruesas y desarenador), se llena al tanque de almacenamiento de 350 litros a través de una bomba de impulsión peristáltica. Posterior a ello se instaló un regulador de caudal para mantener una circulación de agua residual constante al reactor UASB como se observa en la figura 1.

Para el arranque del reactor UASB se utilizó el lodo proveniente del reactor anaerobio de manto de lodos y flujo ascendente de CITRAR-UNI este lodo se tomó a una altura de 1,50 metros del UASB debido a que hay investigaciones asegurando que a dicha altura la eficiencia del lodo es la mejor ${ }^{3}$. El 2 de mayo se inoculó, se añadió agua residual hasta $20 \mathrm{~cm}$ luego se inoculó 7,5 litros de lodo al reactor UASB, luego de esto el reactor UASB se cubrió con plástico negro para evitar el crecimiento de algas al igual que los circuitos de las mangueras. Se inicia el arranque con TRH de 14 horas con un caudal de $35 \mathrm{~mL} / \mathrm{min}$.

\section{Técnicas e instrumentos}

\begin{tabular}{cl}
\hline \multicolumn{1}{c}{ Técnica } & \multicolumn{3}{c}{ Instrumentos } \\
\hline & $\begin{array}{l}\text { Digestor DBR-200 y colorímetro portátil DR-900: para } \\
\text { determinar la DQO. }\end{array}$ \\
& pHmetro digital HI 8424: para determinar el pH y la temperatura \\
Observación & del agua residual. \\
& Termómetro ambiental con base de madera: para determinar la \\
& temperatura del ambiente. \\
& Cinta métrica: para determinar la altura de lodo. \\
\hline
\end{tabular}




\section{RESULTADOS Y DISCUSIÓN}

Se presenta los resultados de las mediciones de los parámetros DQO, sólidos suspendidos totales, temperatura ambiental, temperatura del agua residual, aceites y grasas y $\mathrm{pH}$.

Los tiempos de retención hidráulica reales estudiados dadas las condiciones de campo fueron 14 horas (2 mayo al 20 junio), 12 horas (21 de junio al 13 julio). Como la eficiencia de remoción de materia orgánica disminuía en un TRH de 12 horas es por ello que se retomó el tiempo de retención hidráulico de 14 horas del 27 de julio al 7 de setiembre para encontrar mejores eficiencias de remoción de DQO.

La operación del reactor UASB inició 2 de mayo del 2017, inoculando 7,5 litros de lodo. El tiempo para la aclimatación del lodo inoculado al reactor UASB fue de 30 días.

\section{Comportamiento de la temperatura}

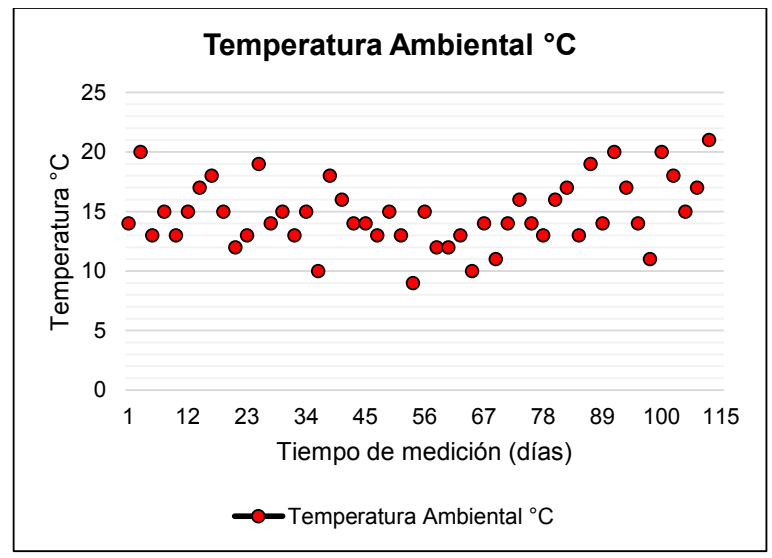

Figura 2. Temperatura ambiental $\left({ }^{\circ} \mathrm{C}\right)$ en función al tiempo acumulado de la investigación (días).

Los resultados de medición de la investigación, muestra la temperatura mínima $9^{\circ} \mathrm{C} \mathrm{y}$ máxima $21^{\circ} \mathrm{C}$, siendo la temperatura ambiental promedio $14,78^{\circ} \mathrm{C}$ en todo el periodo de investigación, el cual fue de 115 días, como se observa en la figura 2. 
Los resultados de la medición de la investigación, muestra la temperatura del agua residual una máxima de $19,9^{\circ} \mathrm{C}$, mínima de $10,3^{\circ} \mathrm{C}$ y promedio de $14,7^{\circ} \mathrm{C}$ en todo el periodo de investigación el cual fue de 115 días, como se observa en la figura 3.

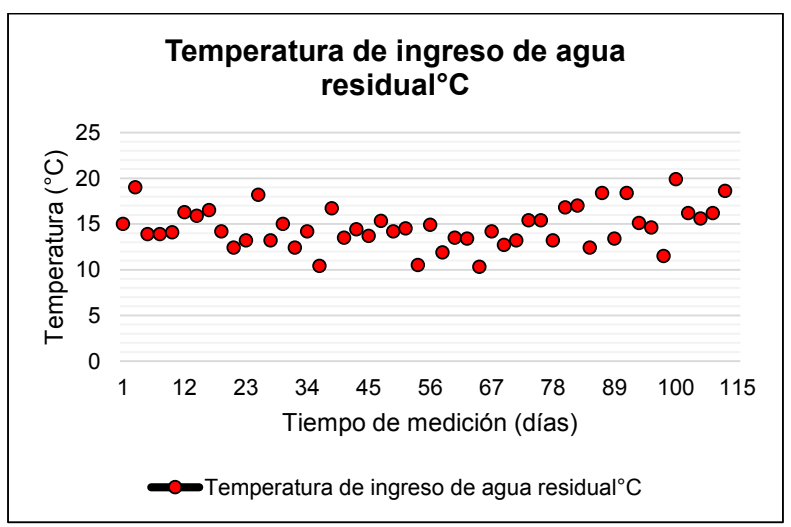

Figura 3. Temperatura de ingreso del agua residual $\left({ }^{\circ} \mathrm{C}\right)$ en función al tiempo acumulado de la investigación (días).

Los resultados de la medición muestran la temperatura del agua residual una máxima de $19,5^{\circ} \mathrm{C}$, mínima de $10,4^{\circ} \mathrm{C}$ y promedio de $14,8^{\circ} \mathrm{C}$ durante los 115 días de la investigación, como se observa en la figura 4 .

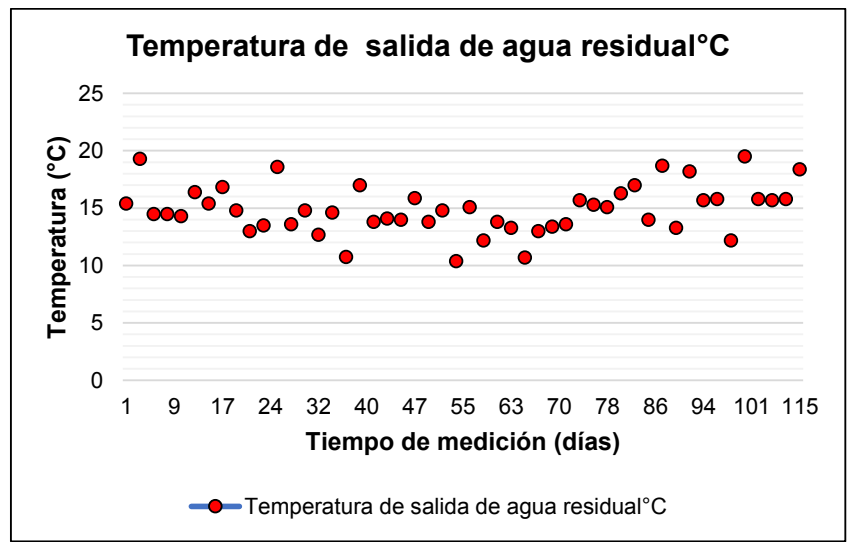

Figura 4. Temperatura de salida del agua residual $\left({ }^{\circ} \mathrm{C}\right)$ en función al tiempo acumulado de la investigación (días).

Si se realiza una comparación de las temperaturas dentro del reactor y la temperatura del ambiente para el día en que se realizó el perfil, se puede observar que la temperatura del reactor se mantuvo en un rango de 20 a $30^{\circ} \mathrm{C}$, mientras que la temperatura del ambiente tuvo rangos de temperatura entre 8 y $32,8^{\circ} \mathrm{C}$, lo que demuestra un rango favorable 4 . 


\section{Comportamiento del potencial de hidrógeno $(\mathrm{pH})$}

Para el punto A (punto de ingreso al reactor UASB) se obtuvo el mínimo valor de $\mathrm{pH} 6,57$, máximo 8,01 y en promedio 7,38; para el punto B (punto de salida del reactor UASB) se obtuvo el mínimo valor de $\mathrm{pH}$ 6,99, máximo 8,32 y en promedio 7,61 durante los 115 días, como se observa en la figura 5.

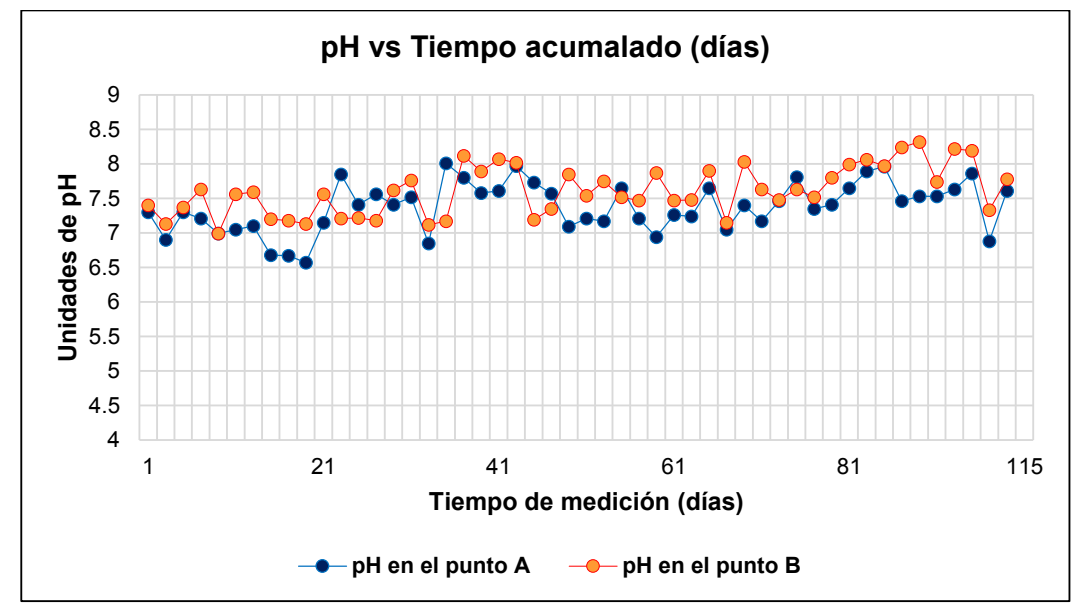

Figura 5. Potencial de hidrógeno promedio $(\mathrm{pH})$ en función al tiempo acumulado de la investigación (días).

El agua residual que ingresa al reactor UASB (Punto A) tiene el pH entre 6,57 y 8,01 favorable para el desarrollo hidrolítico acidogénico y metanogénico. El efluente del reactor UASB (Punto B) tiene el $\mathrm{pH}$ entre 6,99 y 8,32; por lo cual estos resultados de $\mathrm{pH}$ se encuentran óptimos para la digestión anaerobia.

En cuanto al $\mathrm{pH}$, durante el periodo observado, se mantuvo cercano a la neutralidad con un valor de 7,3 $\pm 0,3$. Este valor se encuentra dentro del intervalo recomendado para el mantenimiento de las bacterias metanogénicas. El agua residual de rastro, por la sangre desechada en el proceso, contiene cantidades significativas de proteínas $\mathrm{y}$, al llevarse a cabo el metabolismo de éstas, se produce la liberación de amonio, produciendo bicarbonato de amonio y generando alcalinidad, lo que ayudó a mantener un valor de $\mathrm{pH}$ cercano a 7 en el caso de los dos reactores anaerobios ${ }^{5}$.

\section{Concentración de aceites y grasas}

Se realizaron los análisis en el laboratorio RCJ (LABS UNIVERSAL) de la ciudad de Huancayo el 16/08/2017. Se determinó la concentración de sólidos suspendidos totales para un TRH de 14 horas con un caudal de circulación de $35 \mathrm{~mL} / \mathrm{min}$; tenemos en el punto A una mínima de 522,42 mg/L, máxima de $901,01 \mathrm{mg} / \mathrm{L}$ y en promedio $709,49 \mathrm{mg} / \mathrm{L}$. Para el punto B una mínima de 365,04 mg/L, máxima de $895,05 \mathrm{mg} / \mathrm{L}$ y en promedio $623,76 \mathrm{mg} / \mathrm{L}$. 
En reactores UASB, la formación de nata puede ocurrir en dos lugares distintos: i) al interior del separador trifásico, en la interface de liberación de los gases formados durante la digestión anaerobia; y ii) en la superficie del decantador. Independiente del lugar donde la nata llegue a formarse o acumularse, la cantidad y sus características van a depender, esencialmente, de las características del agua residual, en términos de la presencia de sólidos en suspensión y de la cantidad de aceites y grasas ${ }^{6}$.

\section{Concentración de sólidos suspendidos totales}

Se determinó la concentración de aceites y grasas para un TRH de 14 horas con un caudal de circulación de $35 \mathrm{~mL} / \mathrm{min}$; tenemos en el punto A una mínima de 28,33 mg/L, máxima de $38,02 \mathrm{mg} / \mathrm{L}$ y en promedio 33,32 mg/L. Para el punto B una mínima de 27,09 mg/L, máxima de $28,66 \mathrm{mg} / \mathrm{L}$ y en promedio $27,64 \mathrm{mg} / \mathrm{L}$.

El contenido de sólidos en un agua residual afecta directamente la calidad de lodo que se produce en el sistema de tratamiento o disposición. La eficiencia de remoción de SST en los dos reactores, tuvo un comportamiento variable, puesto que presentó una fluctuación bastante amplia con un máximo valor que supera el $40 \%$ y un mínimo de $2 \%$ en el reactor UASB para temperatura promedio de $17^{\circ} \mathrm{C}^{7}$.

\section{Eficiencias de remoción de demanda química de oxígeno}

Las eficiencias de los parámetros son calculadas en base a los resultados, en A (ingreso de agua residual al reactor UASB) y B (salida de agua residual del reactor UASB). 
Tabla 1. Eficiencias de remoción de DQO evaluados para un tiempo de retención hidráulico de 14 horas en el reactor UASB.

\begin{tabular}{|c|c|c|c|c|}
\hline \multicolumn{5}{|c|}{$\begin{array}{c}\text { EFICIENCIA DE REMOCIÓN DE DQO EN EL } \\
\text { REACTOR UASB }\end{array}$} \\
\hline días & $\begin{array}{c}\text { Caudal } \\
\text { (L/día) } \\
\text { Ingreso a } \\
\text { reactor } \\
\text { UASB }\end{array}$ & $\begin{array}{c}\text { Punto A } \\
\text { (Entrada } \\
\text { al reactor } \\
\text { UASB) } \\
\text { mg/L }\end{array}$ & $\begin{array}{c}\text { Punto B } \\
\text { (Salida } \\
\text { del } \\
\text { reactor } \\
\text { UASB) } \\
\text { mg/L }\end{array}$ & $\begin{array}{c}\text { Eficiencia } \\
\text { de } \\
\text { remoción } \\
\text { DQO }(\%)\end{array}$ \\
\hline $21 / 05 / 17$ & 50 & 780 & 527 & 32,44 \\
\hline $29 / 05 / 17$ & 50 & 963 & 464 & 51,82 \\
\hline $30 / 05 / 17$ & 50 & 715 & 451 & 36,92 \\
\hline $31 / 05 / 17$ & 50 & 777 & 454 & 41,57 \\
\hline $01 / 06 / 17$ & 50 & 588 & 368 & 37,41 \\
\hline $02 / 06 / 17$ & 50 & 1047 & 586 & 44,03 \\
\hline $07 / 06 / 17$ & 50 & 704 & 467 & 33,66 \\
\hline 09/06/17 & 50 & 247 & 63 & 74,49 \\
\hline $10 / 06 / 17$ & 50 & 407 & 94 & 76,90 \\
\hline $13 / 06 / 17$ & 50 & 313 & 120 & 61,66 \\
\hline $14 / 06 / 17$ & 50 & 573 & 235 & 58,99 \\
\hline $15 / 06 / 17$ & 50 & 964 & 343 & 64,42 \\
\hline $20 / 06 / 17$ & 50 & 996 & 460 & 53,82 \\
\hline $13 / 08 / 17$ & 50 & 949 & 589 & 37,93 \\
\hline $16 / 08 / 17$ & 50 & 1120 & 637 & 43,13 \\
\hline $17 / 08 / 17$ & 50 & 1063 & 615 & 42,14 \\
\hline $18 / 08 / 17$ & 50 & 914 & 551 & 39,72 \\
\hline $21 / 08 / 17$ & 50 & 1067 & 678 & 36,46 \\
\hline $22 / 08 / 17$ & 50 & 776 & 402 & 48,20 \\
\hline $23 / 08 / 17$ & 50 & 812 & 441 & 45,69 \\
\hline $25 / 08 / 17$ & 50 & 827 & 449 & 45,71 \\
\hline $28 / 08 / 17$ & 50 & 904 & 394 & 56,42 \\
\hline $07 / 09 / 17$ & 50 & 1044 & 416 & 60,15 \\
\hline \multicolumn{4}{|c|}{ PROMEDIO } & 48,86 \\
\hline \multicolumn{4}{|c|}{ MÁXIMO } & 76,90 \\
\hline \multicolumn{4}{|c|}{ MÍNIMO } & 32,44 \\
\hline
\end{tabular}

La eficiencia de remoción de Demanda Química de Oxígeno (DQO) para un tiempo de retención hidráulico de 14 horas, se obtuvo una mínima de $32,44 \%$, máxima de 76,90 \% y el promedio fue $48,86 \%$, como se observa en la tabla 1 y en la figura 6 . 
Tabla 2. Eficiencias de remoción de DQO evaluados para un tiempo de retención hidráulico de 12 horas en el reactor UASB.

\begin{tabular}{ccccc}
\hline \multicolumn{5}{c}{ EFICIENCIA DE REMOCIÓN DE DQO EN EL } \\
REACTOR UASB \\
\hline $\begin{array}{c}\text { Caudal } \\
\text { (L/día) } \\
\text { Ingreso a } \\
\text { reactor } \\
\text { UASB }\end{array}$ & $\begin{array}{c}\text { Punto A } \\
\text { (Entrada al } \\
\text { reactor } \\
\text { UASB) } \\
\text { mg/L }\end{array}$ & $\begin{array}{c}\text { Punto B } \\
\text { (Salida del } \\
\text { reactor } \\
\text { UASB) } \\
\text { mg/L }\end{array}$ & $\begin{array}{c}\text { Eficiencia } \\
\text { de } \\
\text { remoción } \\
\text { DQO (\%) }\end{array}$ \\
\hline $26 / 06 / 17$ & 58 & 952 & 622 & 34,66 \\
$27 / 06 / 17$ & 58 & 1280 & 874 & 31,72 \\
$29 / 06 / 17$ & 58 & 904 & 595 & 34,18 \\
$30 / 06 / 17$ & 58 & 979 & 618 & 36,87 \\
$04 / 07 / 17$ & 58 & 949 & 585 & 38,36 \\
$06 / 07 / 17$ & 58 & 1057 & 643 & 39,17 \\
$07 / 07 / 17$ & 58 & 1083 & 646 & 40,35 \\
$10 / 07 / 17$ & 58 & 929 & 537 & 42,20 \\
$11 / 07 / 17$ & 58 & 988 & 576 & 41,70 \\
$13 / 08 / 17$ & 58 & 849 & 501 & 40,99 \\
\hline \multicolumn{5}{c}{ PROMEDIO } \\
MÁXIMO & & $\mathbf{3 8 , 0 2}$ \\
MÍNIMO & $\mathbf{4 2 , 2 0}$ \\
\hline \multicolumn{5}{c}{}
\end{tabular}

La eficiencia de remoción de Demanda Química de Oxigeno (DQO) para un tiempo de retención hidráulico de 12 horas, se obtuvo una mínima de $31,72 \%$, máxima de $42,20 \%$ y el promedio fue $38,02 \%$, como se observa en la tabla 2 y en la figura 7 .

Asimismo, de las pruebas realizadas en el estudio, se puede afirmar que para una temperatura del agua residual de 10 a $19{ }^{\circ} \mathrm{C}$ en el reactor UASB, al evaluar el TRH de 14 horas, por 92 días, se obtuvo un porcentaje de remoción de DQO máxima de 76,90\% y la mínima fue de $32,44 \%$. Posteriormente, se disminuyó el TRH a 12 horas, por 23 días, el porcentaje de DQO máxima fue de $42,20 \%$ y la mínima fue de $31,72 \%$.

Según Seghezzo se utilizaron reactores anaeróbicos para temperaturas $7-18^{\circ} \mathrm{C}$ con tiempo de retención hidráulico de 4-14 horas. Por ello la investigación empezó con el arranque del reactor UASB, el 2 de mayo del 2017 con un TRH (tiempo de retención hidráulico) de 14 horas hasta el 20 de junio, del 21 de junio al 13 de julio se continuó con tiempo de retención hidráulico de 12 horas. Como la eficiencia de remoción de materia orgánica disminuía en un TRH de 12 horas es por ello que se retomó el tiempo de retención hidráulico de 14 horas del 27 de julio al 7 de setiembre para encontrar mejores eficiencias de remoción de DQO.

Los resultados de la baja eficiencia de remoción de DQO es debido al efecto de las bajas temperaturas del ambiente, como también podría deberse a que el TRH con el que se opera es muy corto como para permitir un tiempo apropiado de contacto entre la materia orgánica y los microorganismos que la consumen. También se debe prolongar el periodo de arranque del reactor UASB, ya que en la presente investigación se tuvo un periodo de arranque de 30 días. 
La remoción de materia orgánica expresada como DQO se evaluó para tres TRH: 36, 24 y 12 horas. Al evaluar el TRH de $36 \mathrm{~h}$, por 28 días, se obtuvo un porcentaje de remoción de DQO promedio de $88 \%$. Posteriormente, se disminuyó el TRH a $24 \mathrm{~h}$, el porcentaje de DQO promedio fue de $87 \%$. Finalmente, se evaluó por 22 días el reactor para un TRH de $12 \mathrm{~h}$ y una CO (carga orgánica) de $17 \mathrm{~kg} / \mathrm{m} 3 \mathrm{~d}$, obteniéndose un porcentaje de remoción de DQO promedio de $79 \%$, estos resultados se presentaron a una temperatura de $37^{\circ} \mathrm{C}^{8}$.

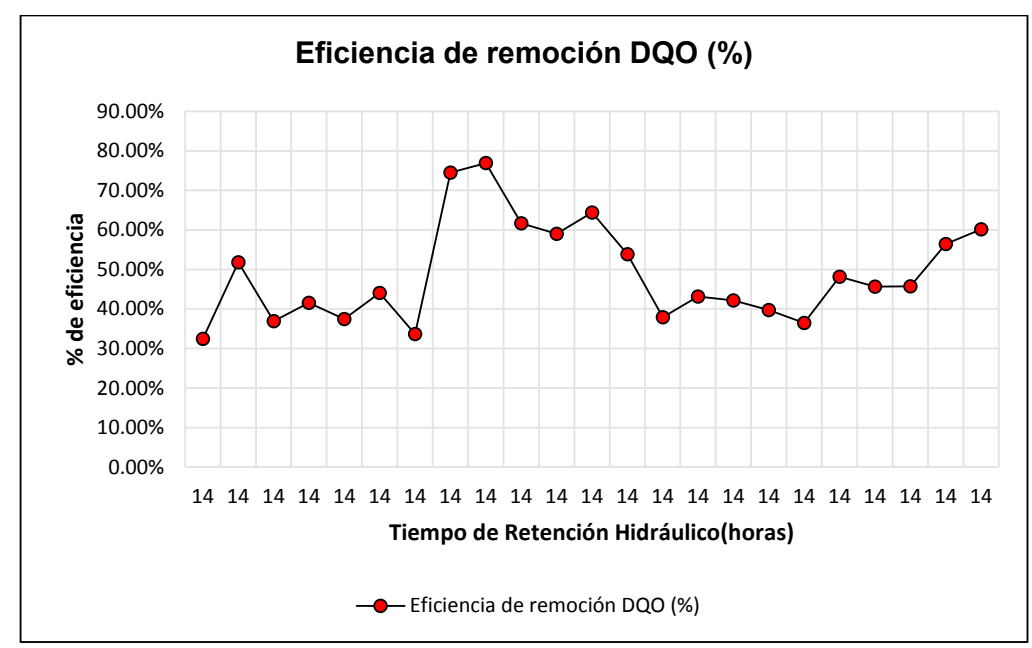

Figura 6. Eficiencia de remoción de DQO en el reactor UASB para un TRH de 14 horas.

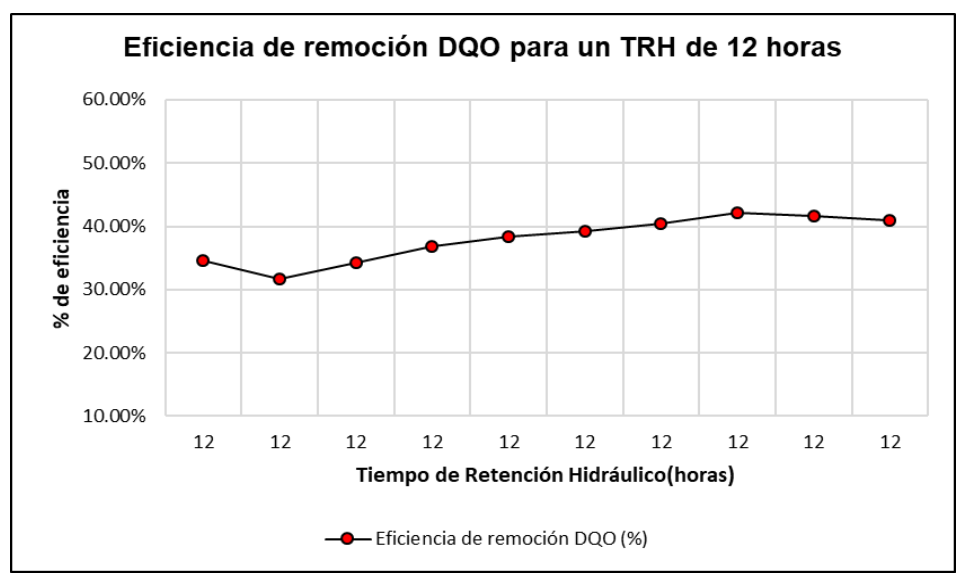

Figura 7. Eficiencia de remoción de DQO en el reactor UASB para un TRH de 12 horas. 
De esta forma, se observa el tendiente creciente de la eficiencia del reactor UASB en la medida que se aumenta el tiempo de retención hidráulico.

Los resultados indican que con cuatro TRH de operación $(14,12,10$ y 8 horas) la remoción de DQO total sobrepasa el $49 \%$, siendo el mejor resultado para un TRH de 14 horas con $60,7 \%$ de remoción promedio de DQO total, la más baja remoción resultó para un TRH de 4 horas con 25,3\% de remoción promedio de DQO total, todo ello fue con un digestor de lodo 9 .

A bajas temperaturas $\left(6-16^{\circ} \mathrm{C}\right)$ el reactor debe operarse a una VCO (carga orgánica volumétrica) máxima de $0,6 \mathrm{kgDQO} / \mathrm{m}^{3} \mathrm{~d}$, ya que en esta condición se logra obtener una buena remoción de materia orgánica total y particulada, mientras que al operar con VCO (carga orgánica volumétrica) mayores disminuye notoriamente la eficiencia del reactor ${ }^{10}$.

\section{Altura de lodo}

La altura de lodo del UASB está condicionado por el crecimiento de la biomasa y por la velocidad ascensional aplicado ambos originan su crecimiento, el crecimiento por biomasa es lento, en los cuatro meses de operación creció el lodo de $42 \mathrm{~cm}$ al inicio del arranque del reactor UASB a $76 \mathrm{~cm}$ al finalizar la investigación con una velocidad ascensional de $0,12 \mathrm{~m} / \mathrm{h}$ y $0,14 \mathrm{~m} / \mathrm{h}$, no se purgó del reactor UASB debido a que el crecimiento por biomasa es lento.

La expansión del lodo es producida por la velocidad ascensional variada por cada TRH. Se aprecia que hay una relación directamente proporcional de la altura de lodo a la velocidad ascensional aplicado, la altura de lodo en un $96 \%$ es afectado por la velocidad ascensional, el porcentaje que hace falta para el $100 \%$, probablemente es por el crecimiento biológico de biomasa en el reactor UASB. Entonces, la velocidad ascensional es un parámetro clave en la expansión de lodo del reactor UASB, se facilita también su expansión por las características floculentas del lodo'.

El reactor UASB operando con aguas residuales urbanas no puede operar a Vs (velocidad de sedimentación) de $0,7 \mathrm{~m} / \mathrm{h}$ ya que se produce el lavado de biomasa y expansión de la cama de lodos disminuyendo la eficiencia global de remoción y la de materia orgánica debida a sólidos suspendidos. A Vs (velocidad de sedimentación) de $0,5 \mathrm{~m} / \mathrm{h}$ se logra mantener una concentración de biomasa adecuada en el reactor y esta biomasa prevalece en forma granular, aunque presenta bordes más irregulares ${ }^{10}$.

\section{CONCLUSIONES}

En la investigación se obtuvieron óptimas remociones de materia orgánica, lo cual es medida como DQO, para un tiempo de retención hidráulico de 14 horas se obtuvo una eficiencia de remoción máxima de 76,90 \% de DQO y un promedio de 48,86 \% de DQO, como se observa en la tabla 1. Para un tiempo de retención hidráulico de 12 horas se obtuvo una eficiencia de remoción máxima de $42,20 \%$ de DQO y un promedio de 38,02 \% de DQO, como se observa en la tabla 2. Lo cual se concluye que el reactor anaerobio de manto de lodos de 
flujo ascendente (UASB) como tratamiento primario remueve la materia orgánica del agua residual del Camal Municipal de Huancavelica.

Se evaluó la concentración de sólidos suspendidos totales para un TRH de 14 horas con un caudal de circulación de $35 \mathrm{~mL} / \mathrm{min}$; tenemos en la entrada al reactor UASB una mínima de $522,42 \mathrm{mg} / \mathrm{L}$ y una máxima de $901,01 \mathrm{mg} / \mathrm{L}$. A la salida del reactor mínima de 365,04 mg/L y una máxima de $895,05 \mathrm{mg} / \mathrm{L}$. Se concluye que el reactor UASB influye en la baja producción de lodo debido a la digestión anaerobia.

$\mathrm{El}$ agua residual en el reactor UASB tuvo un $\mathrm{pH}$ dentro del rango de 6,57 y 8,32. Se concluye que el agua residual que ingresa al reactor UASB tiene el $\mathrm{pH}$ favorable para el desarrollo hidrolítico acidogénico y metanogénico. Por lo cual estos resultados de $\mathrm{pH}$ se encuentran en los rangos entre 6,50 y 8 óptimos para la digestión anaerobia ${ }^{6}$.

El reactor UASB fue capaz de mantener una temperatura promedio $14,89{ }^{\circ} \mathrm{C}$ siempre por encima de la temperatura ambiental que tuvo una temperatura promedio de $14,79{ }^{\circ} \mathrm{C}$. Para una temperatura del agua residual de 10 a $19{ }^{\circ} \mathrm{C}$ y manteniendo un tiempo de retención hidráulico de 14 horas en el reactor UASB, se han logrado valores máximos de remoción de $76,90 \%$ en DQO.

\section{AGRADECIMIENTO}

Agradecimiento al Instituto de Investigación de la Universidad Nacional de Huancavelica por el apoyo brindado durante la realización de esta investigación. Nuestro agradecimiento al Ing. Yon Cecilio Cabrera por el apoyo incondicional a la presente investigación.

\section{REFERENCIAS BIBLIOGRÁFICAS}

1. Chaux G, Rojas G, Bolaños L. Producción más limpia y viabilidad de tratamiento biológico para efluentes de mataderos en pequeñas localidades Caso: Municipio de El Tambo (Colombia). Rev Bio Agro. 2009; 7(1): 102-114.

2. Caldera Y, Madueño P, Griborio A, Fernández N, Gutiérrez E. Effect of the organic load in the performance the UASB reactor treating slaughterhouse effluent. [Internet]. Rev Téc Fac Ing Univ Zulia. Agosto de 2005; 28(2). [citado 15 jun 2019]. Disponible en: http://ve.scielo.org/scielo.php?script=sci_arttext\&pid=S0254-07702005000200003.

3. De la cruz Martínez LA, Orellana Rivas V. Caracterización del desagüe industrial de la pasta de pota para la remoción de carga orgánica utilizando un sistema combinado de UASB y lodos activados. [Tesis de grado] Lima, Perú: Universidad Nacional de Ingeniería; 2011.

4. García CC. Evaluación de la capacidad de remoción de materia orgánica de las aguas residuales de los rastros en reactor UASB. [Tesis de maestría]. Morelia: Universidad Michoacana de San Nicolás de Hidalgo; 2012. 
5. Reyes MD. Tratamiento de aguas residuales provenientes de rastro mediante un sistema de biodegradación anaerobia - aerobia. [Tesis de maestría] México: Universidad Nacional Autónoma de México; 2009.

6. De lemos CA. Reactores anaerobios. Principios del tratamiento biológico de aguas residuales. Pasto (Colombia): Universidad de Nariño; 2013.

7. Sierra Ramírez DC, Vivas Albarracín C. Diseño e implementación de una unidad piloto de tratamiento biológico no convencional para los vertimientos generados en el matadero de macanal (Corpochivor). [Tesis de grado]. Bógota (Colombia): Universidad de la Salle; 2005.

8. Caldera Y. The effect of hydraulic retention time in the functioning fthe USAB reactor in slaughterhouse effluent treatment. Multiciencias. 2003; 3(1):33-40.

9. Quispe JA. Estudio de la remoción de carga orgánica en un reactor anaerobio de manto de lodos de flujo ascendente con digestor de lodos aplicado a una localidad ubicado a una altitud de $3800 \mathrm{msnm}$. [Tesis de grado]. Lima (Perú): Universidad Nacional de Ingeniería; 2014.

10. Gonzáles P, Chamy R, Poirrier P. Tratamiento anaerobio de aguas residuales urbanas mediante un reactor UASB en clima templado frío. [Internet]. Valparaíso: Pontificia Universidad Católica de Valparaíso; 2000. [Citado 13 mayo 2019]. Disponible en: https://pdfs.semanticscholar.org/38eb/ 792aa1b906026068d58d0d53616365aea986.pdf 